\title{
Frequency dependent polarization analysis of ambient seismic noise recorded at a broadband seismometer in the central United States*
}

\author{
Keith D. Koper ${ }^{1, \star}$ and Veronica L. Hawley ${ }^{2}$ \\ ${ }^{1}$ Department of Geology and Geophysics, University of Utah, Salt Lake City, Utah 84112, USA \\ ${ }^{2}$ Department of Earth and Atmospheric Sciences, Saint Louis University, St. Louis, MO 63108, USA
}

\begin{abstract}
We present a new approach to polarization analysis of seismic noise recorded by three-component seismometers. It is based on statistical analysis of frequency-dependent particle motion properties determined from a large number of time windows via eigenanalysis of the 3-by-3, Hermitian, spectral covariance matrix. We applied the algorithm to continuous data recorded in 2009 by the seismic station SLM, located in central North America. A rich variety of noise sources was observed. At low frequencies $(<0.05 \mathrm{~Hz})$ we observed a tilt-related signal that showed some elliptical motion in the horizontal plane. In the microseism band of $0.05-0.25 \mathrm{~Hz}$, we observed Rayleigh energy arriving from the northeast, but with three distinct peaks instead of the classic single and double frequency peaks. At intermediate frequencies of $0.5-2.0 \mathrm{~Hz}$, the noise was dominated by non-fundamental-mode Rayleigh energy, most likely P and Lg waves. At the highest frequencies $(>3 \mathrm{~Hz})$, Rayleigh-type energy was again dominant in the form of $\mathrm{Rg}$ waves created by nearby cultural activities. Analysis of the time dependence of noise power shows that a frequency range of at least $0.02-1.0 \mathrm{~Hz}$ (much larger than the microseism band) is sensitive to annual, meteorologically induced sources of noise.
\end{abstract}

Key words: seismic noise; polarization analysis; central North America

CLC number: P315.3 Document code: A

\section{Introduction}

Analysis of ambient seismic noise is becoming increasingly relevant to modern seismology. Advances in computational speed and storage have made it feasible to analyze years and even decades of continuous seismic data in short amounts of time. Therefore, it is now possible to perform longitudinal studies of station performance in order to identify degradation or mis-installation of seismic equipment (e.g., www.ldeo.columbia.edu/ $\sim$ ekstrom/Projects/WQC.html). Long-term noise analysis also provides insight into the evolution of the ocean wave climate, specifically whether the frequency and intensity of storms have changed as global temperatures have changed (Bromirski et al., 1999; Grevemeyer et al., 2000; Aster et al., 2008, 2010; Koper et al., 2009).

\footnotetext{
* Received 7 June 2010; accepted in revised form 3 August 2010; published 10 October 2010.

* Corresponding author. e-mail: kkoper@gmail.com

(c) The Seismological Society of China and Springer-Verlag Berlin Heidelberg 2010
}

The most straightforward method of analyzing seismic noise is calculation of the power spectrum of seismograms from which the instrument response has been removed. Individual spectra can be averaged over long time periods, eliminating the influence of transient signals, to create 2D histograms, or probability density functions (PDFs), that reveal the strength of the background noise as a function of frequency (McNamara and Buland, 2004). The PDFs can be compared to reference noise curves (Peterson, 1993) to classify the detection quality of the seismometer as a function of frequency. Time variations of the spectra can also be examined to determine which frequencies possess daily or yearly periodicities, and more fundamentally, to determine time periods in which the instrument was malfunctioning or the instrument response was incorrect. This type of processing is carried out routinely for thousands of seismometers that transmit data to the IRIS DMC (Incorporated Research Institutions for Seismology Data Management Center) and results are publicly available on the web (www.iris.edu/quack). 
More sophisticated processing of seismic noise is possible if an array of seismometers is available. In this case, the 2D slowness vector of the background noise can be directly estimated. Phase velocity can be used to identify the propagation mode of the noise and the backazimuth can be used to identify its source region (e.g., Koper and de Foy, 2008; Koper et al., 2009). Temporal variations in the locations of ambient noise sources can also be closely tracked with array data (e.g., Gerstoft et al., 2006). Unfortunately, arrays have useful slowness resolution only over a relatively small range of frequencies for which the wavefield is properly sampled by the sensor geometry. Many permanent arrays, such as those used by the International Monitoring System (IMS), are designed to detect high-frequency energy $(>1$ $\mathrm{Hz}$ ), and have apertures that are too small to be used for studies in the microseismic band of about $0.05-0.25 \mathrm{~Hz}$.

An alternative method of analyzing seismic noise that is effective over a wide range of frequencies, yet provides more insight than simple power spectra, is polarization analysis (e.g., Schulte-Pelkum et al., 2004; Tanimoto et al., 2006). When ground motion is recorded by three-component sensors, the phase relationships among the components are strong indicators of the propagation mode of the ambient noise, for instance whether it is a body wave, Rayleigh wave, or Love wave. The orientation of the polarization ellipsoid also constrains the backazimuth and incidence angle of the incoming energy. Directional constraints from polarization analysis are generally weaker than those obtained from array analysis (e.g., Harris, 1990), but they have the advantage of being observable over a wide range of frequencies.

In this work, we describe and implement a novel approach to polarization analysis of ambient seismic noise using data recorded by a broadband seismometer (SLM) located in central North America (Figure 1). We process one calendar year of SLM data to identify coherent structures in the noise field across three orders of magnitude in frequency $(0.01-10 \mathrm{~Hz})$. This particular station was selected, in part, because it is installed at a site that has been continuously occupied for over a century, with analog SLM records dating back to 1906 archived at Saint Louis University. These data may be useful as a proxy for the evolution of the ocean wave climate in the northern hemisphere over this time period. Plans are being made to digitize these data for a longitudinal noise study, but as a first step it is necessary to obtain a thorough understanding of the present day

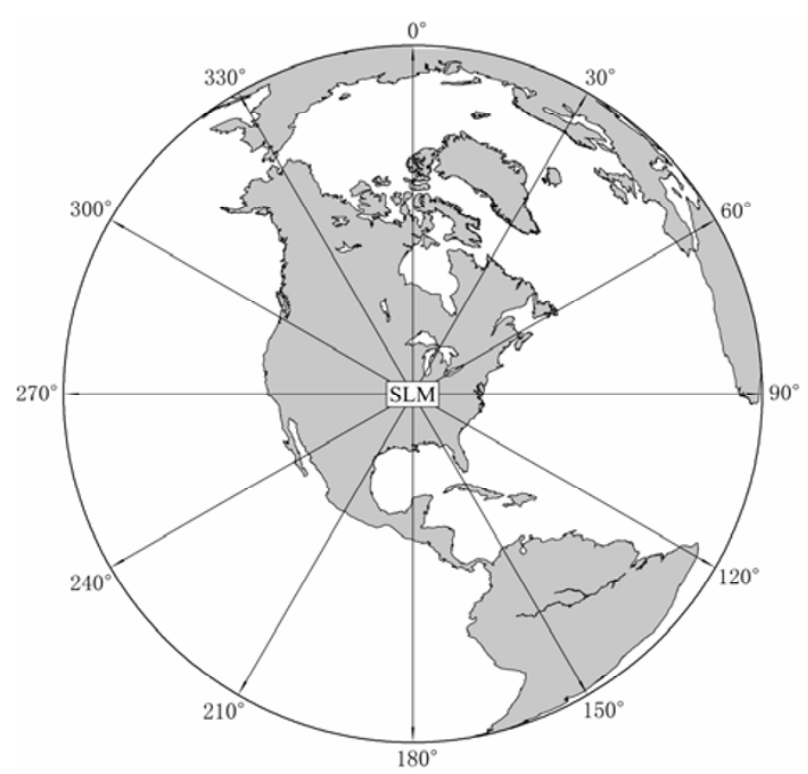

Figure 1 Location of seismometer SLM. The map projection is orthographic and shows the incoming azimuth of potential microseismic source locations. The plot border occurs at a distance of 90 degrees.

ambient noise field at SLM.

\section{Methodology}

A variety of related techniques have been developed for performing polarization analysis of three-component seismic data (e.g., Kanasewich, 1973; Vidale, 1986; Jurkevics, 1988; Bataille and Chiu, 1991). The technique used here is loosely based on the work of Samson (1983), as described by Park et al. (1987), and is illustrated with a flow chart in Figure 2. Essentially, eigen-decomposition of the spectral covariance matrix associated with a sliding window of data is applied to yield various polarization attributes as a function of time and frequency. This in turn yields fundamental information about the composition of seismic noise, such as the extent to which it is polarized, its mode of propagation, and the direction from which it arrives at the seismometer.

The processing begins by extracting one hour of data from each broadband component and removing the instrument response. A typical segment of data is shown in Figure 3, along with the response of the seismograph system to ground acceleration. Each window is divided into 16 subwindows of 8192 points (409.6 s), which overlap one another by $50 \%$. Each subwindow is detrended and tapered with a Hanning function, Fourier transforms are calculated, and the elements of the spectral covariance matrix are calculated. The diagonal ele- 
ments of the matrix represent the power spectra of each component, while the off-diagonal elements represent cross-spectra among the three components. The matrix is $3-$ by-3 and Hermitian, and its elements are functions of frequency, which ranges from $0 \mathrm{~Hz}$ to $10 \mathrm{~Hz}$ in increments of $2.441 \times 10^{-3} \mathrm{~Hz}$. The sixteen covariance matrices are linearly averaged to provide an overall covariance matrix for the original one hour segment of data. Eigen-decomposition is performed and the principal eigenvalue and eigenvector are assumed to represent the polarization characteristics of the noise over the one hour period.
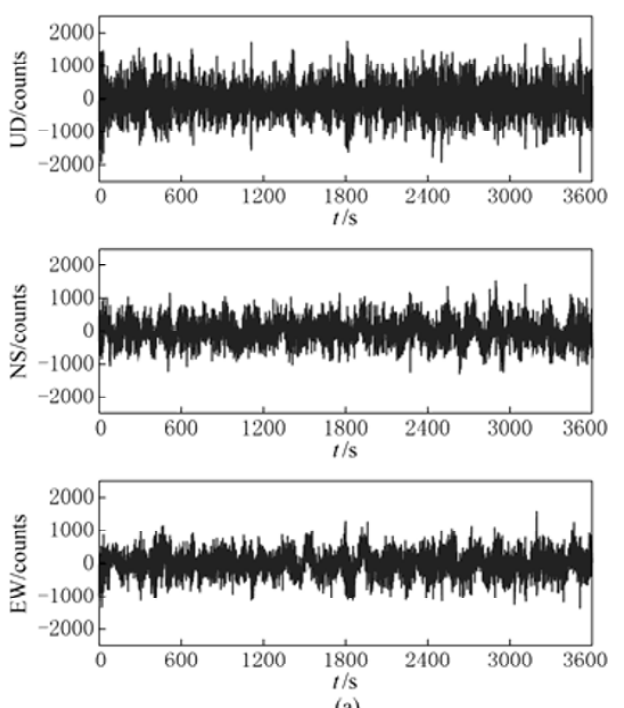

(a)

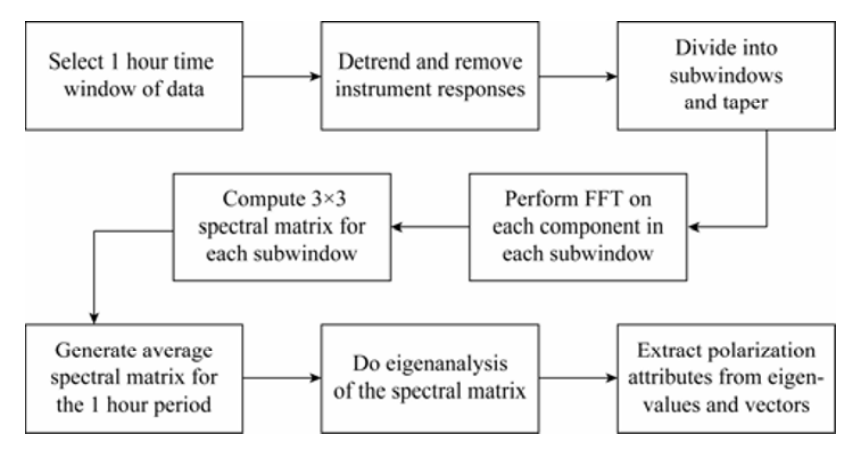

Figure 2 Flow chart for polarization analysis of ambient noise recorded at SLM. Details are given in the text.

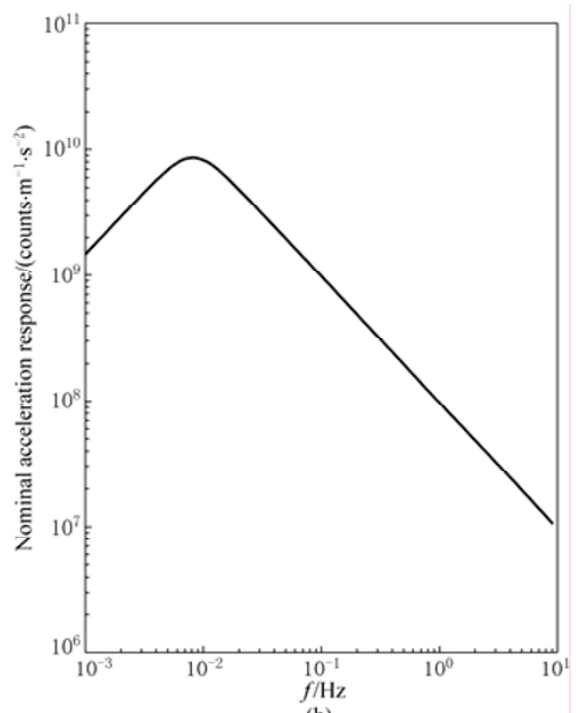

(b)

Figure 3 (a) Seismograms recorded at SLM during the second hour of January 1, 2009. The only processing was trend removal. (b) Nominal response of the SLM recording system. Gains are not appreciably different for the three components.

Output from this procedure is presented in Figure 4 for the example seismograms shown in Figure 3. The three panels on the left, Figures 4a, 4b, 4c, show power spectra for the vertical (UD), east-west (EW), and north-south (NS) components respectively. Each has the same general appearance with a broad peak in noise power at very low frequencies $(<0.01 \mathrm{~Hz})$, three sharp peaks within the microseism band $(0.05-0.25 \mathrm{~Hz})$, and a broad peak at very high frequencies $(>3 \mathrm{~Hz})$. The dropoff in amplitude near $8 \mathrm{~Hz}$ is an artifact related to the anti-aliasing FIR filters in the data logger. The corresponding power spectrum for the primary eigenvalue $(\lambda)$ is shown in Figure 4d. Its variation as a function of frequency is similar to that of the individual components.

The degree of polarization $\left(\beta^{2}\right)$ is presented in Figure 4e. It is defined by Samson (1983) to measure the extent to which the noise is organized and can be described by fewer than three degrees of freedom. It varies from 0 , when all the eigenvalues are equal, to 1 when only a single non-zero eigenvalue exists. For the three-component recordings considered here it is defined as

$$
\beta^{2}=\frac{3 t\left(\boldsymbol{S}^{2}\right)-[t(\boldsymbol{S})]^{2}}{2[t(\boldsymbol{S})]^{2}}
$$

where $t$ is the trace and $\boldsymbol{S}$ is spectral covariance matrix.

We emphasize that the eigenvalues in this study are derived differently from some time-domain polarization analyses (e.g., Jurkevics, 1988) and that a large value of $\beta^{2}$ does not imply linear particle motion. Elliptical motion can also lead to high values of $\beta^{2}$. As a function of frequency, $\beta^{2}$ generally tracks the variation in primary 
eigenvalue power, though some differences exist.

In Figures $4 \mathrm{f}, 4 \mathrm{~g}, 4 \mathrm{~h}, 4 \mathrm{i}$ we present the frequency dependence of four angular quantities $\left(\Theta_{\mathrm{H}}, \Theta_{\mathrm{V}}, \phi_{\mathrm{VH}}, \phi_{\mathrm{HH}}\right)$ derived from the eigenvector associated with the primary eigenvalue. The first two quantities relate to the orientation of the polarization ellipsoid, with $\Theta_{\mathrm{H}}$ representing backazimuth for a Rayleigh or $\mathrm{P}$ wave (degrees clockwise from North), and $\Theta_{\mathrm{V}}$ representing angle of incidence for a $\mathrm{P}$ wave. The latter two quantities indicate phase relations among the components, with $\phi_{\mathrm{VH}}$ being the difference in phase between the vertical and principal horizontal component, and $\phi_{\mathrm{HH}}$ being the difference in phase between the two horizontal components. Details on calculating these quantities are given in Park et al. (1987).
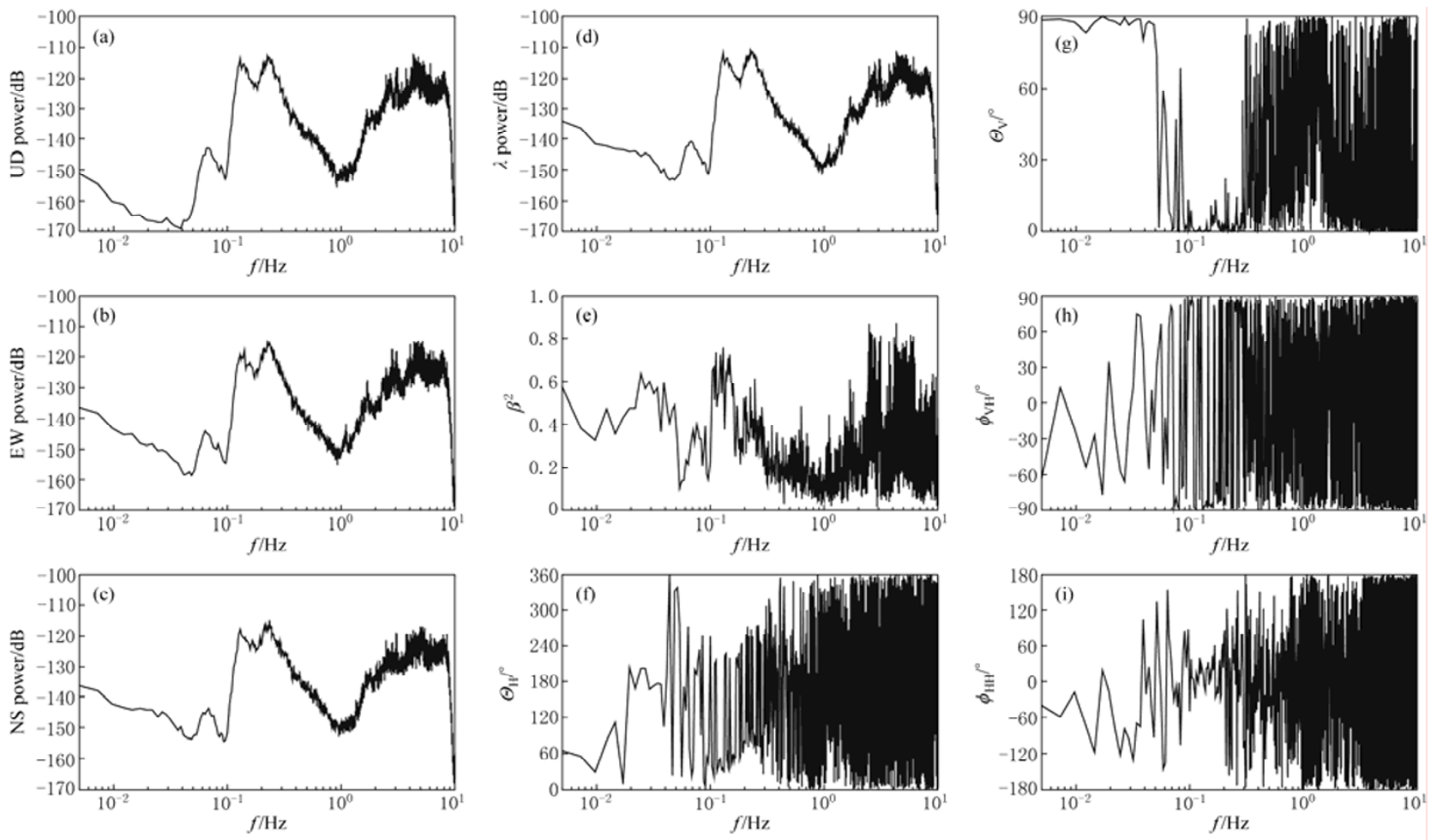

Figure 4 Results from polarization analysis of the seismograms shown in Figure 3. Measures of power as a function of frequency are shown in units of $10 \log _{10}\left(\mathrm{~m}^{2} \mathrm{~s}^{-4} \mathrm{~Hz}^{-1}\right)$ for the vertical component (a), the east-west component (b), the north-south component (c), and the principal eigenvalue $\lambda(\mathrm{d})$. The degree of polarization $\left(\beta^{2}\right)$ shown in (e) is dimensionless. The azimuth $\left(\Theta_{\mathrm{H}}\right)$ and inclination $\left(\Theta_{\mathrm{V}}\right)$ of the polarization ellipsoid are shown in panels $(\mathrm{f})$ and $(\mathrm{g})$ respectively, in units of degrees. Phase differences between the vertical and principal horizontal components $\left(\phi_{\mathrm{VH}}\right)(\mathrm{h})$ and the two horizontal components $\left(\phi_{\mathrm{HH}}\right)$ (i) are also presented in degrees.

The directional parameters $\Theta_{\mathrm{H}}$ and $\Theta_{\mathrm{V}}$ are not well defined for strongly elliptical particle motion and Park et al. (1987) recommend caution in interpreting them when either $\phi_{\mathrm{VH}}$ or $\phi_{\mathrm{HH}}$, respectively, is within $20^{\circ}$ of $\pm 90^{\circ}$. A second problematic issue is that the polarization ellipsoid can "tip over", such that a retrograde Rayleigh wave from a particular direction can appear as a prograde Rayleigh wave from the opposite direction. This can cause a $180^{\circ}$ ambiguity in the arrival direction $\left(\Theta_{\mathrm{H}}\right)$ of a Rayleigh wave.

Some structure is evident in the angular quantities presented in Figure 4 such as high $\Theta_{\mathrm{V}}$ for frequencies less than $0.05 \mathrm{~Hz}$ and low $\Theta_{\mathrm{V}}$ for frequencies of about
0.1-0.3 Hz; however, in general there are rapid fluctuations as a function of frequency. These are probably created by the low overall degree of polarization, and the tipping ambiguity described previously.

\section{Results}

\subsection{Polarization characteristics of SLM noise}

Because of the erratic nature of polarization quantities measured from individual windows of weakly polarized noise (Figure 4 is typical), it is more meaningful to make inferences about the noise field at SLM from a statistical analysis of large numbers of measurements. We present this in Figure 5, in which the polarization 
attributes extracted from all available hours of data in 2009 are binned into PDFs in a manner analogous to that described in McNamara and Buland (2004). This figure shows that although the noise is often weakly polarized, there are organized features that exist in sev- eral distinct frequency bands. We note that because of the extensive time averaging, the influence of transient signals from earthquakes and explosions is negligible in Figure 5, while quasi-stationary signals from ocean waves, wind, traffic, etc., are enhanced.
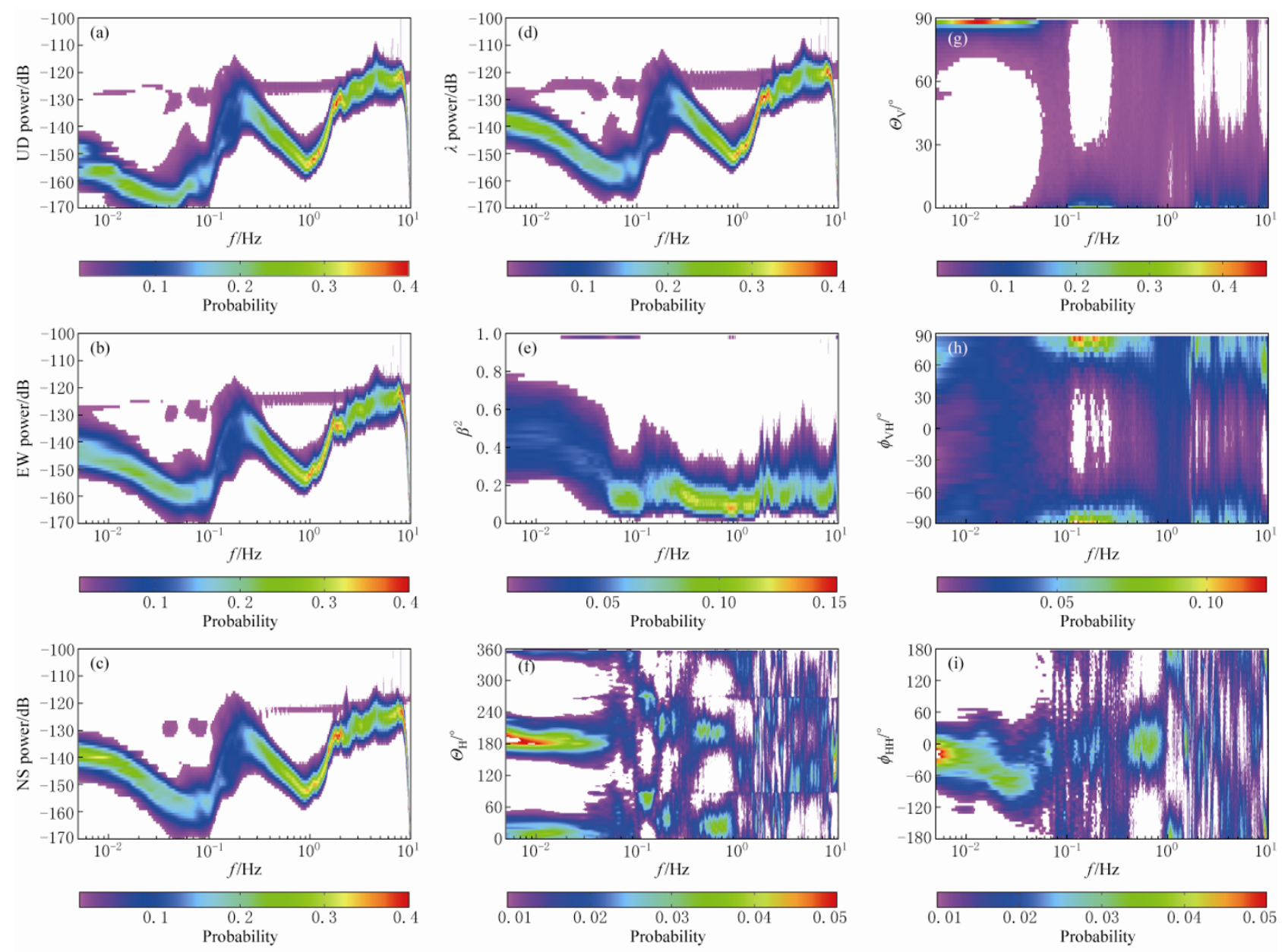

Figure 5 Probability density functions (PDFs) for the power and polarization characteristics of ambient noise recorded at SLM during 2009. Each frequency index is individually normalized so that integration over the entire range of the dependent variable gives unity. Panels are as defined in Figure 4.

At the lowest frequencies $(<0.05 \mathrm{~Hz})$ the noise is highly polarized, leading to peaked PDFs for the angular quantities. Examining the binned power spectra of the individual components (Figures 5a, 5b, 5c) it is clear that horizontal motion dominates over vertical, causing the $\Theta_{V}$ PDFs to peak sharply near $90^{\circ}$. It is also clear that north-south power is significantly stronger than east-west power, causing the $\Theta_{\mathrm{H}} \mathrm{PDFs}$ to cluster tightly near $0^{\circ}$ and $180^{\circ}$. The $\phi_{\mathrm{VH}}$ PDFs are very broad, probably because of the insignificance of vertical motion. In contrast, the $\phi_{\mathrm{HH}} \mathrm{PDFs}$ are peaked near values of $0^{\circ}-60^{\circ}$, implying some amount of horizontal elliptical motion.
We interpret this energy to be composed primarily of ground tilt near the recording site.

In the microseismic band $(0.05-0.3 \mathrm{~Hz})$ there are three distinct noise peaks, as suggested by the individual sample shown in Figure 4. The weakest of these occurs near $0.075 \mathrm{~Hz}$ as indicated by small bumps in the power spectra (though there is no corresponding bump in $\beta^{2}$ ). This energy is strongly elliptical in the vertical-radial plane, with $\phi_{\mathrm{VH}}$ PDFs clustered around $\pm 90^{\circ}$. The backazimuths for this band are weakly clustered around $45^{\circ}-60^{\circ}$, as well as the exact opposite direction $\left(225^{\circ}-\right.$ $\left.240^{\circ}\right)$. For retrograde arrivals $\left(\phi_{\mathrm{VH}}>0\right)$ the backazimuths 
tend towards the northeast, as opposed to the southwest, and we interpret this energy to be typical, retrograde Rayleigh energy arriving from the northeast.

The second peak in the microseism band occurs at $0.13 \mathrm{~Hz}$ and is clearly distinguished from a third peak near $0.20 \mathrm{~Hz}$ by an abrupt change in backazimuth. In the power spectra PDFs, the two peaks are less distinct, presumably because of smearing related to slight variations in the dominant frequencies of the microseismic sources over time. Polarization is increased for these peaks relative to the $0.075 \mathrm{~Hz}$ peak, and Rayleigh propagation is indicated by the clustering of $\phi_{\mathrm{VH}}$ near $\pm 90^{\circ}$ and $\phi_{\mathrm{HH}}$ near $0^{\circ}$. This implies that while $\Theta_{\mathrm{V}}$ lacks meaning, $\Theta_{\mathrm{H}}$ is informative. A $180^{\circ}$ ambiguity exists in backazimuth, but as before, the retrograde arrivals preferentially arrive from the northeast.

Continuing to the higher frequencies, there are at least two more distinct regimes. At frequencies of about $0.5-2.0 \mathrm{~Hz}$ the horizontal motion is linear with $\phi_{\mathrm{VH}}$ clustered around either $0^{\circ}$ or $\pm 180^{\circ}$, leading to well defined $\Theta_{\mathrm{H}}$ PDFs, aligned NE-SW as in the previous cases. However, the PDFs for $\phi_{\mathrm{VH}}$ are broadly diffuse, leading to poorly defined values of $\Theta_{\mathrm{V}}$. This energy is clearly not fundamental-mode Rayleigh waves, and is probably a mixture of $\mathrm{P}$ and $\mathrm{Lg}$ waves, which are known to dominate in this frequency band (Koper et al., 2010). At the highest frequencies $(>3 \mathrm{~Hz})$ the noise reverts back to Rayleigh, or $\mathrm{Rg}$, propagation as indicated by the values of $\phi_{\mathrm{NH}}$ and $\phi_{\mathrm{HH}}$. The backazimuths are scattered, probably implying multiple nearby sources of cultural noise.

\subsection{Time dependence of SLM noise}

Additional insight into the nature of the ambient noise field at SLM can be gained by inspecting the time dependence of the noise power. We present this in Figure 6 for seven frequency bands that sample the various noise regimes described in the previous section $(0.02 \mathrm{~Hz}$, $0.075 \mathrm{~Hz}, 0.2 \mathrm{~Hz}, 0.6 \mathrm{~Hz}, 1.0 \mathrm{~Hz}, 2.0 \mathrm{~Hz}$, and $5.0 \mathrm{~Hz}$ ). In each case the power of the principal eigenvalue is

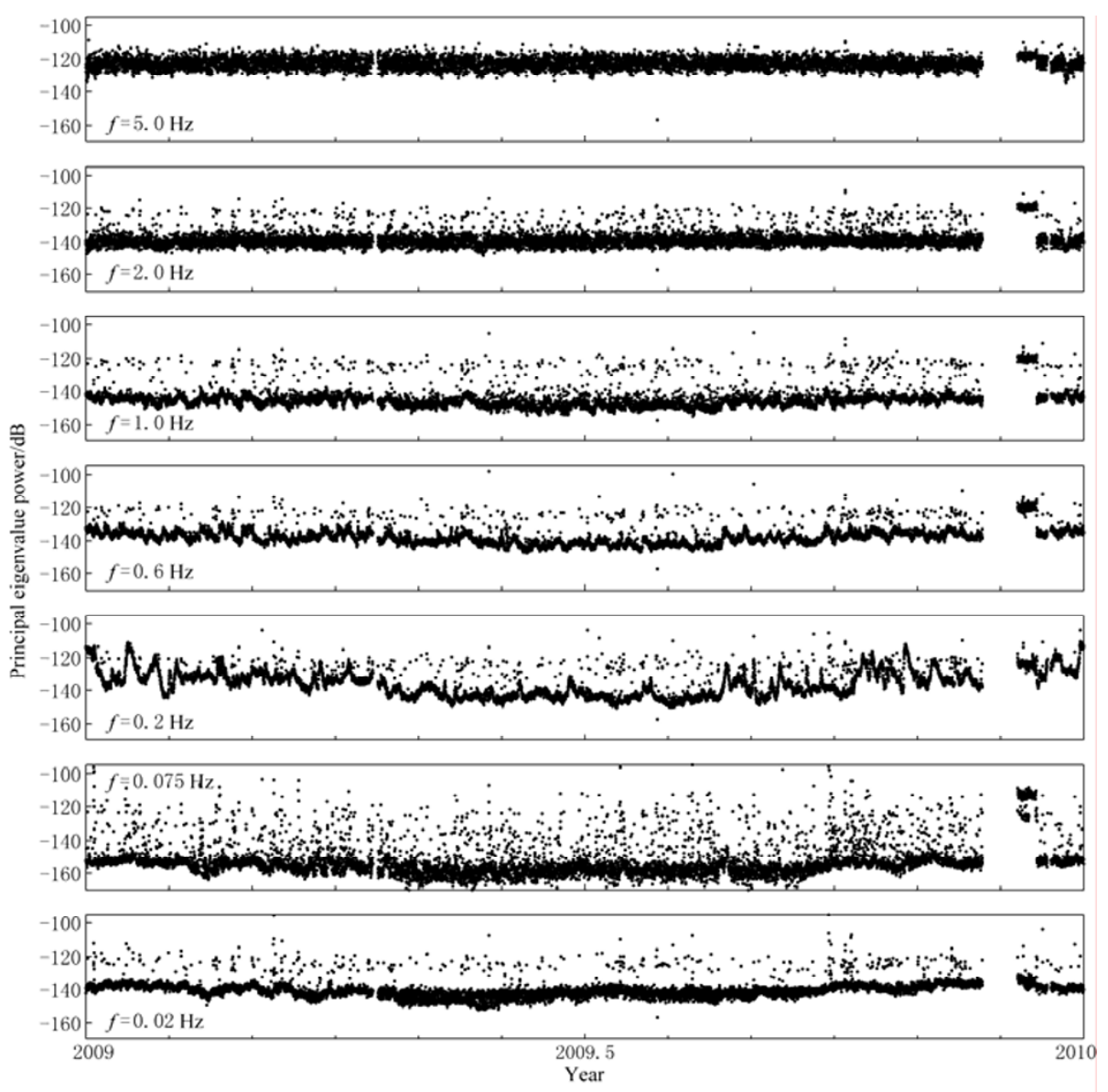

Figure 6 Power of the principal eigenvalue for seven selected frequency bands (center frequency of $f_{i}$ ), in units of $10 \log _{10}\left(\mathrm{~m}^{2} \mathrm{~s}^{-4} \mathrm{~Hz}^{-1}\right)$. In each case, power is $f_{i}$ averaged in the frequency domain between $f_{i} / \sqrt{2}$ and $\sqrt{2} f_{i}$. Time is in fractional years for 2009, with tick marks placed every $1 / 12$ of the year. 
averaged between $f / \sqrt{2}$ and $\sqrt{2} f$ for each hourly sample of noise. The excursions from the baseline trends are due mainly to earthquakes. The data gap late in the year, and the distorted amplitudes immediately following it, were caused by telemetry problems transmitting the data to the U.S. National Earthquake Information Center, from which it was extracted for this study.

Many of the frequency bands show lower noise levels in the months of May, June, July, and August, as expected for microseisms generated by wave action in the northern hemisphere, as well as a diurnal signal that peaks during daylight hours, as expected for cultural noise such as road traffic. We quantify this by computing periodograms for the time series shown in Figure 6. Each time series is groomed with a deglitching algorithm that replaces apparent earthquake signals with the yearly median. Data gaps are also filled with the yearly median, the trend is removed, and the number of points is padded with zeros until 16384 prior to computing the FFT. No subwindowing or tapers are applied.

The resulting periodograms are presented in Figure 7. Each is individually normalized. We experimented with different methods of deglitching and found that only frequencies higher than about $2-3 \mathrm{~d}^{-1}$ were significantly affected. The power at lower frequencies is insensitive to the details of the spectral operations. The frequency bands from $0.02 \mathrm{~Hz}$ through $1.0 \mathrm{~Hz}$ all show strong annual signals, even though only a single cycle is sampled. This is not caused by spectral leakage from lower frequencies since the trend (including the mean) was removed prior to the FFT. It is noteworthy that this natural, meteorologically related variation is observable far outside the traditional microseismic band of $0.05-$ $0.25 \mathrm{~Hz}$.
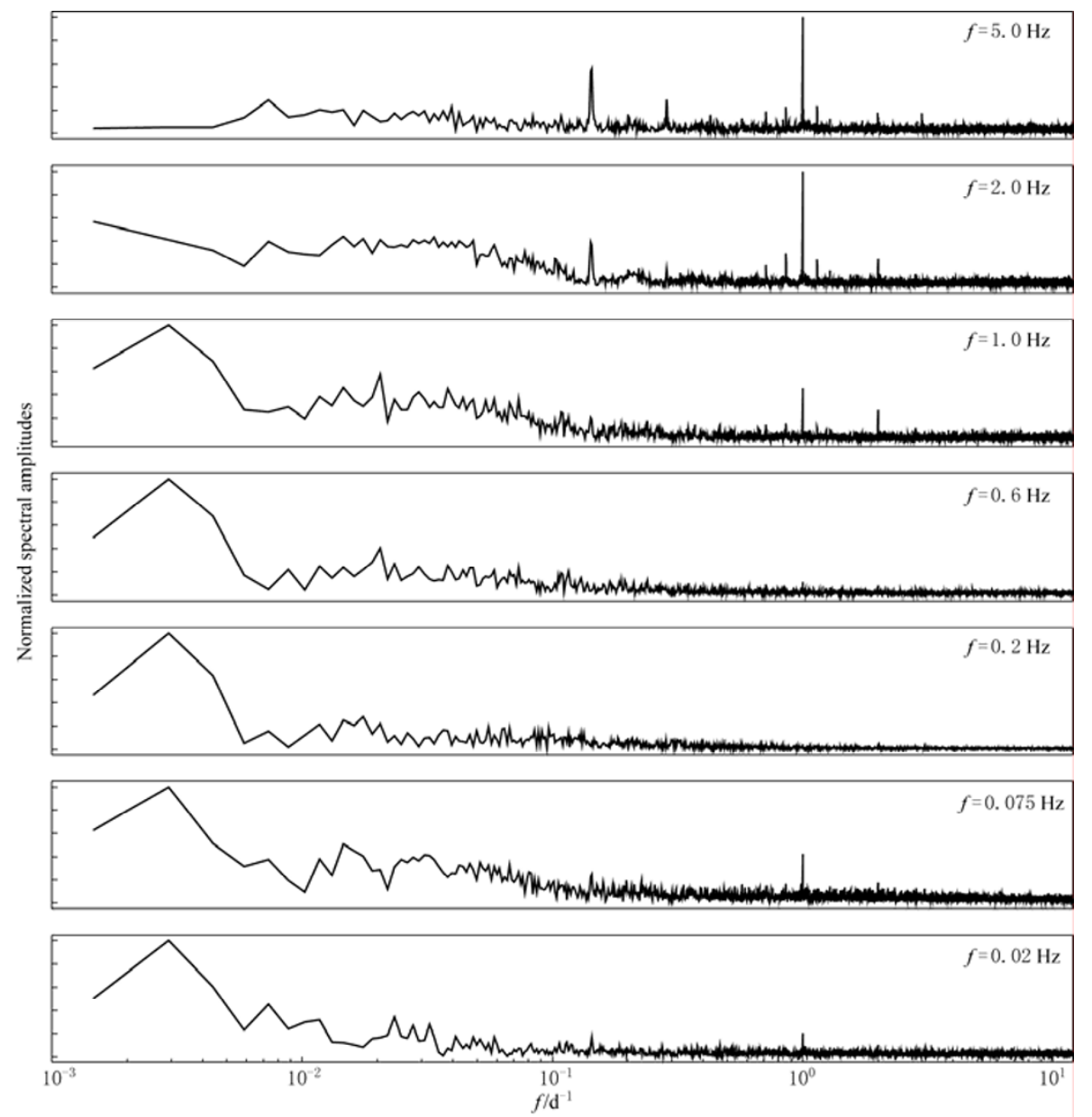

Figure 7 Periodograms of the noise trends shown in Figure 6.

A strong diurnal peak, and some higher harmonics are observed for the highest frequency bands centered at
$1.0 \mathrm{~Hz}, 2.0 \mathrm{~Hz}$, and $5.0 \mathrm{~Hz}$, as well as for the two lowest frequency bands centered at $0.02 \mathrm{~Hz}$ and $0.075 \mathrm{~Hz}$. The 
higher frequency diurnal peaks are almost certainly related to cultural activities since they also show significant energy at weekly frequencies, with decreased power during weekends. This is expected since SLM is located in an urban environment. The cause of the diurnal peak at the lower frequencies is more uncertain. Both periodograms show a hint of power at weekly frequencies which would seem to imply a cultural source; however, it is not obvious what particular cultural source would operate at such a low frequency. On the other hand, the ground tilt we infer to be occurring at low frequencies could plausibly be influenced by day-night temperature variations, leading to a diurnal signal.

\section{Conclusions}

We developed and implemented a new approach to studying seismic noise recorded at three component seismometers. It is an extension of the method of McNamara and Buland (2004) and uses cross-spectral information to infer the polarization characteristics of ambient seismic noise. We applied the algorithm to data recorded in 2009 by the seismometer SLM in central North America. Several distinct noise regimes were discovered including a tilt signal at low frequencies $(<0.05 \mathrm{~Hz})$, fundamental-mode Rayleigh waves at microseismic frequencies $(0.05-0.25 \mathrm{~Hz}), \mathrm{P}$ and $\mathrm{Lg}$ waves at intermediate frequencies $(0.5-2.0 \mathrm{~Hz})$, and $\mathrm{Rg}$ waves at microtremor frequencies $(>3.0 \mathrm{~Hz})$.

The Rayleigh waves in the microseism band arrived from the northeast and were probably generated by storm activity in the North Atlantic, a region known to be a strong generator of microseismic energy (e.g., Kedar et al., 2008); however, from the work presented here it is unclear if the energy is generated along the coastline or in deep water. Interestingly, there seems to be three distinct noise sources in this band with peaks at $0.075 \mathrm{~Hz}, 0.013 \mathrm{~Hz}$, and $0.20 \mathrm{~Hz}$, instead of the expected two peaks. The middle peak arrives from a distinctly different direction $\left(\sim \mathrm{N} 60^{\circ} \mathrm{E}\right)$ than the other two $\left(\sim \mathrm{N} 30^{\circ} \mathrm{E}\right)$, implying at least two separate source regions.

Strong diurnal variation is observed at low frequencies $(<0.075 \mathrm{~Hz})$ and high frequencies $(>1.0 \mathrm{~Hz})$, with stronger power during the local daytime. The highfrequency noise source is almost certainly cultural since these frequency bands also show weekly periodicities, with reduced noise on the weekends. The low-frequency source is most likely natural and related to day-night temperature differences that influence ground tilt near the sensor; however, we cannot rule out a cultural source based solely on the observations.

Acknowledgments We thank Bob Herrmann for discussions about seismic equipment installed at SLM, and Dan McNamara for discussions about power spectra. Many of the figures were created with GMT (Wessel and Smith, 1991). This work was partially supported by the United States Geological Survey.

\section{References}

Aster R C, McNamara D E and Bromirski P D (2008). Multidecadal climate-induced variability in microseisms. Seism Res Lett 79: 194-202.

Aster R C, McNamara D E and Bromirski P D (2010). Global trends in extremal microseism intensity. Geophys Res Lett 37: L14303, doi:10.1029/2010GL043472.

Bataille K and Chiu J M (1991). Polarization analysis of high-frequency, three-component seismic data. Bull Seismol Soc Am 81: 622-642.

Bromirski P D, Flick R E and Graham N (1999). Ocean wave height determined from inland seismometer data: Implications for investigating wave climate changes in NE Pacific. $J$ Geophys Res 104: 20 753-20 766.

Gerstoft P, Fehler M C and Sabra K G (2006). When Katrina hit California. Geophys Res Lett 33: L17308, doi:10.1029/2006 GL027270.

Grevemeyer I, Herber R and Essen H (2000). Microseismological evidence for a changing wave climate in the northeast Atlantic Ocean. Nature 408: 349-352.

Harris D (1990). Comparison of the direction estimation performance of high-frequency seismic arrays and three-component stations. Bull Seismol Soc Am 80: 1 951-1 968.

Jurkevics A (1988). Polarization analysis of three-component array data. Bull Seismol Soc Am 78: 1 725-1 743.

Kanasewich E R (1973). Time Sequence Analysis in Geophysics. The University of Alberta Press, Edmonton, 274-296.

Kedar S, Longuet-Higgins M, Webb F, Graham N, Clayton R and Jones C (2008). The origin of deep ocean microseisms in the North Atlantic Ocean. Proc R Soc A 464: 777-793.

Koper K D and de Foy B (2008). Seasonal anisotropy in short-period seismic noise recorded in South Asia. Bull Seismol Soc Am 98: 3 033-3 045.

Koper K D, de Foy B and Benz H (2009). Composition and variation of noise recorded at the Yellowknife Seismic Array, 1991-2007. J Geophys Res 114: B10310, doi:10.1029/2009 JB006307.

Koper K D, Seats K and Benz H M (2010). On the composition of Earth's short period seismic noise field. Bull Seismol Soc Am 100: 606-617.

McNamara D E and Buland R P (2004). Ambient noise levels in the continental United States. Bull Seismol Soc Am 94: 1 5171527. 
Park J, Vernon F L and Lindberg C R (1987). Frequency dependent polarization analysis of high-frequency seismograms. J Geophys Res 92: 12 664-12 674.

Peterson J (1993). Observation and modeling of seismic background noise. USGS Open File Report 93-322. United Stated Geological Survey, Reston, VA, 1-42.

Samson J C (1983). Pure states, polarized waves, and principal components in the spectra of multiple, geophysical time-series. Geophys J R astr Soc 72: 647-664.
Schulte-Pelkum V, Earle P S and Vernon F L (2004). Strong directivity of ocean-generated seismic noise. Geochem Geophys Geosys 5: Q03004, doi:10.1029/2003GC000520.

Tanimoto T, Ishimaru S and Alvizuri C (2006). Seasonality in particle motion in microseisms. Geophys J Int 166: 253-266.

Vidale J E (1986). Complex polarization analysis of particle motion. Bull Seismol Soc Am 76: 1 393-1 405.

Wessel P and Smith W (1991). Free software helps map and display data. EOS Trans $A G U$ 72: 441, 445-446. 